\title{
Application of two lines of chemotherapy: gemcitabine with nab-paclitaxel and liposomal irinotecan with 5 -fluorouracil and leucovorin in patient with advanced pancreatic adenocarcinoma
}

\author{
Krystyna Ostrowska-Cichocka' ${ }^{1}$ Marek Z. Wojtukiewicz ${ }^{1,2}$ \\ 'Department of Clinical Oncology with the Subdivision of Daily Chemotherapy, Dr. Ewa Pilecka \\ Memorial Bialystok Cancer Center \\ ${ }^{2}$ Department of Oncology, Medical University of Bialystok
}

Correspondence:

Krystyna Ostrowska-Cichocka Department of Clinical Oncology with the Subdivision of Daily Chemotherapy,

Dr. Ewa Pilecka Memorial

Bialystok Cancer Center

15-027 Białystok, ul. Ogrodowa 12

phone: (85) 6646714

Received:

8.02.2021

Accepted:

10.07.2021

DOI: 10.24292/01.OR.123100721

Copyright $\odot$ Medical Education.

All rights reserved.

\section{ABSTRACT}

Pancreatic cancer is a disease with high mortality. It is predicted to become the second leading cause of cancer death in some regions of the world. Frequent diagnosis at an advanced stage contributes to 5 -year survival at a highly unsatisfactory level of $2-9 \%$. Due to the lack of early symptoms, nearly $80 \%$ of patients receive a diagnosis when distant metastases develop. So far, the most frequently used programs in the treatment of patients with pancreatic cancer in the stage of neoplastic spreading include: FOLFIRINOX (oxaliplatin, 5-fluorouracil, irinotecan, leucovorin), gemcitabine alone or in combination with nab-paclitaxel or erlotinib.

Based on the results of the phase III study NAPOLI-1, liposomal irinotecan in combination with 5-fluorouracil (5-FU) and leucovorin (LV) was approved as the first regimen for use in the second or subsequent line of therapy in patients with advanced pancreatic cancer previously treated with gemcitabine. This paper presents a case of a patient with advanced pancreatic cancer who was treated with two lines of chemotherapy - gemcitabine in combination with nab-paclitaxel and liposomal irinotecan with 5-FU/LV. The treatment was well tolerated and was considered a valuable therapeutic option. A 2-year overall survival was obtained from the diagnosis of the disease.

Key words: advanced pancreatic cancer, gemcitabine in combination with nab-paclitaxel, liposomal irinotecan with 5-FU/LV 


\section{INTRODUCTION}

Pancreatic cancer is one of the most common causes of cancer death in the world. Its incidence shows an upward trend, and it is worth noting that the highest incidence and number of deaths are found in developed countries. In Poland, pancreatic cancer accounts for about $2.5 \%$ of cancer cases and is responsible for $4 \%$ of cancer deaths in men and $6 \%$ in women. In statistics, it ranks fourth-sixth among the causes of deaths from malignant neoplasms. It is estimated that only $5 \%$ of patients survive 5 years from diagnosis, and the morbidity and mortality rates are similar [1-4]. The prognosis for this group of patients is unfavorable.

Pancreatic cancer patients are treated by a specialized multidisciplinary team consisting of gastroenterologists, surgeons, clinical oncologists, radiotherapists, radiologists and specialists in palliative medicine [4]. In most patients the disease is diagnosed in the inoperable stage, then the only possible treatment is palliative chemotherapy. The ineffectiveness of most treatment programs also contributes to the poor prognosis. Currently, there are several treatment regimens in patients with advanced pancreatic cancer: FOLFIRINOX, 5-FU/LV, gemcitabine as monotherapy or in combination with erlotinib, and gemcitabine with nab-paclitaxel [5]. The choice of the type of chemotherapy depends primarily on the patient's performance status, the presence of comorbidities as well as the toxicity of the treatment and its acceptance by the patient [6].

According to the guidelines of the European Society for Medical Oncology (ESMO), the American Society of Clinical Oncology (ASCO) and the National Comprehensive Cancer Network (NCCN) in routine practice, the FOLFIRINOX regimen (phase III PRODIGE4/ACCORD study-11) is recommended as a standard in patients with metastatic pancreatic cancer with the performance level (ECOG, PS) 0-1, without significant comorbidities, with normal or nearly normal bilirubin levels $[7,8]$. The combination of gemcitabine and nab-paclitaxel (MPACT phase III study) is an option for people not eligible for treatment with the FOLFIRINOX regimen $[1,2,9,10]$. The results of the above studies showed the benefit of using multi-drug chemotherapy in terms of overall survival (OS) in patients with advanced pancreatic cancer compared to the earlier standard - gemcitabine [11].

In the case of disease progression after I line chemotherapy approximately $40-50 \%$ of patients with advanced pancreatic cancer may be qualified for second-line chemotherapy and subsequent lines. There is no specific standard of therapy for patients with pancreatic cancer with disease progression after I line treatment. In a systematic review of the second-line treatment options, combination therapies compared with monotherapy resulted in an extension of progression-free survival (PFS) (2.5 vs 1.9 months), but not OS (5.1 vs 4.3 month) [6].

Patients with disease progression following FOLFIRINOX I line treatment may be eligible for treatment with gemcitabine, e.g. in combination with nab-paclitaxel (non-randomized data) or gemcitabine alone. In patients who received gemcitabine in the I line, regimens including 5-FU (FOLFOX-4 or OFF) should be used. A clinical benefit was obtained as a result of the use of such therapy in terms of PFS and OS (PFS - 2.9 months, OS 5.7 months) $[2,4]$.

Another drug registered in the second-line treatment of patients with metastatic pancreatic adenocarcinoma is liposomal irinotecan. It is used in combination with 5-FU/LV in adult patients with disease progression following treatment with gemcitabine. In a randomized phase III study (NAPOLI-1), liposomal irinotecan in combination with 5-FU/LV significantly extended the median OS compared to 5 -FU (6.1 vs 4.2 months) $[12,13]$.

\section{CASE REPORT}

A 63-year-old patient who was treated 20 years ago for Hodgkin's disease, which resulted in dilated cardiomyopathy with paroxysmal atrial fibrillation, with an ejection fraction (EF) of 36\%, was admitted to the Department of Clinical Oncology of the Bialystok Cancer Center in Bialystok due to pancreatic cancer on September $6^{\text {th }}, 2018$. The patient complained of abdominal pains for several months, he lost about $15 \mathrm{~kg}$. A computed tomography (CT) examination of the abdominal cavity, performed on July $28^{\text {th }}$, 2018 , revealed a tumor of the pancreas with a size of $43 \times 36 \times$ $34 \mathrm{~mm}$, with the possibility of an infiltration of the back wall of the stomach and the splenic vein causing its obstruction. In addition, numerous wide veins of collateral circulation have been described around the curvature of the stomach. There were no enlarged nodes in the abdominal cavity or in the peritoneal space. The liver was not enlarged on CT examination, with no focal lesions. Tumor markers remained normal (CEA - $2.4 \mathrm{ng} / \mathrm{ml}$ and CA $19-9-15.0 \mathrm{U} / \mathrm{ml}$ ). The patient was operated on August $8^{\text {th }}$, 2018. Peripheral pancreatectomy and splenectomy were performed, and a liver lesion that could be a metastasis was excised. The histopathological examination revealed the G2pT2 adenocarcinoma (lesion diameter $2.7 \mathrm{~cm}$ ), the presence of neoplastic cells in the cut-off line of the pancreatic tumor, no metastases in the lymph nodes of the splenic hilum and in the surrounding 
tissue of pancreas, while in the tumor removed from the liver, adenocarcinoma was found. Magnetic resonance imaging (MRI) of the abdominal cavity after surgery performed on September $4^{\text {th }}$, 2018 , showed no changes with suspected metastases.

The patient was offered treatment with gemcitabine as monotherapy due to the high risk of cardiological complications after consulting with cardiologist. Three courses of chemotherapy were given from September to November 2018; the therapy was well tolerated. From the next course, gemcitabine was added to a reduced dose of nab-paclitaxel (a gift from the drug manufacturer), registered in combination with gemcitabine as a I line drug in adults with metastatic pancreatic adenocarcinoma.

Due to the lack of measurable changes, the patient could not be qualified for the National Health Fund (NHF, pol. Narodowy Fundusz Zdrowia) drug program. The treatment was well tolerated. Clinical improvement was achieved. The side effects, G3 neutropenia was seen which necessitated the use of short-acting growth factors - administration of filgrastim (granulocyte-colony stimulating factor [G-CSF]). The imaging examinations: $\mathrm{CT}$, MRI, positron emission tomography/computed tomography (PET/CT) showed no evidence of tumor progression. The treatment was completed in May 2019. The patient remained in a very good general condition. Cardiological parameters did not deteriorate.

During subsequent follow-up examinations in September 2020, a $12 \mathrm{~mm}$ hypodense area with blurred outlines appeared in the abdominal CT in the stump of the pancreas, slightly heterogeneously strengthening after the administration of contrast, suggesting a cystic lesion after treatment or another process. The PET/CT examination performed on October $3^{\text {rd }}, 2019$ revealed a recurrence of the neoplastic process within the head of the pancreas on the border with the surgical site after the pancreatic body and tail.

The patient was referred to the Department of Oncological Gastroenterology of the Oncology Center M. Sklodowskiej-Curie in Warsaw to consider stereotaxic radiotherapy. On November $4^{\text {th }}$, 2019, an endoscopic ultrasound (EUS) examination showed a $28 \times 21 \mathrm{~mm}$ tumor in the head and isthmus of the pancreas with the possibility of portal vein infiltration. The available parts of the liver revealed at least three hypoechoic focal lesions ranging in size from 8 to $12 \mathrm{~mm}$. A biopsy of the liver lesion and the pancreatic isthmus tumor were performed through the stomach wall. Markers were also introduced into the pancreatic tumor before planned radiotherapy. The presence of neoplastic cells in both the pancreatic tumor and the liver lesion was confirmed. Abdominal CT scan (fig. 1) as part of the treatment planning confirmed multiple liver metastases. Radiotherapy was abandoned. The CEA tumor marker concentration increased to $809 \mathrm{ng} / \mathrm{ml}$, CA 19-9 concentration remained within the normal range - $26.95 \mathrm{U} / \mathrm{ml}$. There was an increased concentration of CRP $100.3 \mathrm{mg} / \mathrm{l}$, bilirubin - $2.88 \mathrm{mg} / \mathrm{dl}$, ALP - $278 \mathrm{U} / \mathrm{l}$ (results from November $\left.30^{\text {th }}, 2020\right)$, as well as an increase in the concentration of D-dimers - from $16251 \mathrm{ng} / \mathrm{ml}$ November 28 $8^{\text {th }}, 2019$ to $79,403 \mathrm{ng} / \mathrm{ml}$ December $1^{\text {st }}, 2019$, thrombocytopenia 91 000/ $\mathrm{mm}^{3}$ and increase in ALT activity - $160 \mathrm{U} / \mathrm{l}$, AST - $69 \mathrm{U} / \mathrm{I}$. It was noted that the abdominal CT scan from November $29^{\text {th }}$, 2019 described the suspicion of thrombus in the inferior vena cava at the exit of the renal veins. The Doppler ultrasound of the veins of the lower extremities and the inferior vena cava showed no evidence of inferior vena cava thrombosis. It was decided to include therapeutic doses of low-molecular-weight heparin, which allowed for a significant clinical improvement and an improvement in blood biochemical parameters, with particular emphasis on the parameters of the coagulation and hepatic systems. At that time, the patient complained of periodic pain in the abdomen. Fentanyl and oral morphine were included which enabled good pain control.

FIGURE 1.

Computed tomography of the abdominal cavity (liver) on November $29^{\text {th }}, 2019$.

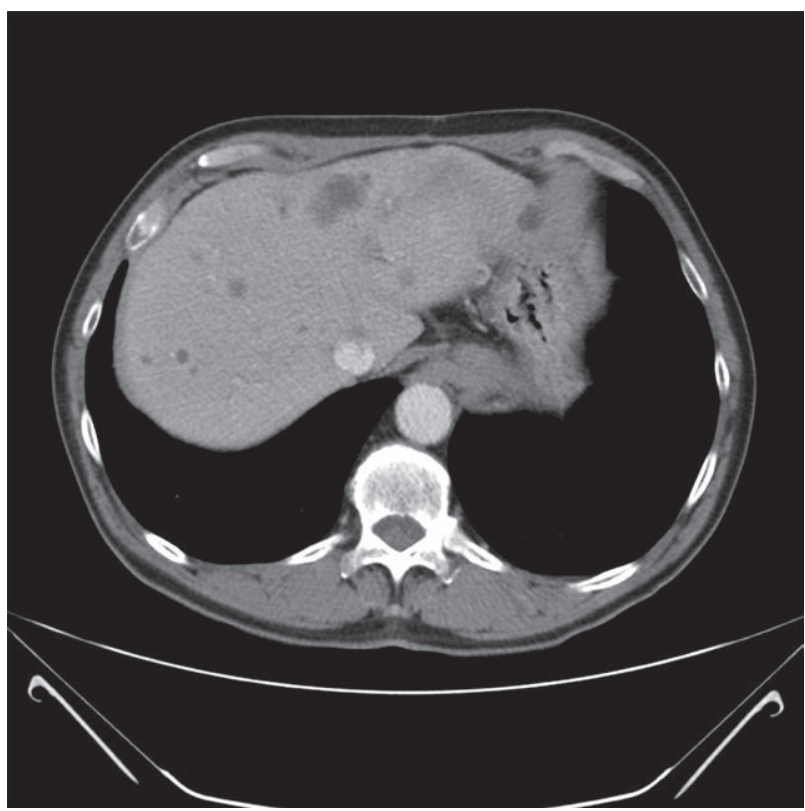


FIGURE 2.

Computed tomography of the abdominal cavity (liver) on June $5^{\text {th }}$, 2020.

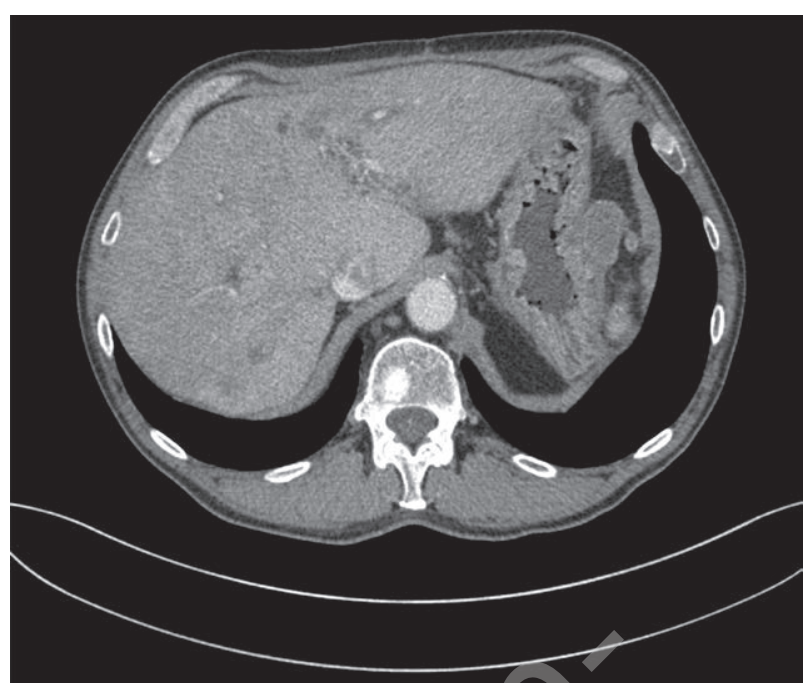

FIGURE 3.

Magnetic resonance imaging of the central nervous system on June $25^{\text {th }}, 2020$.

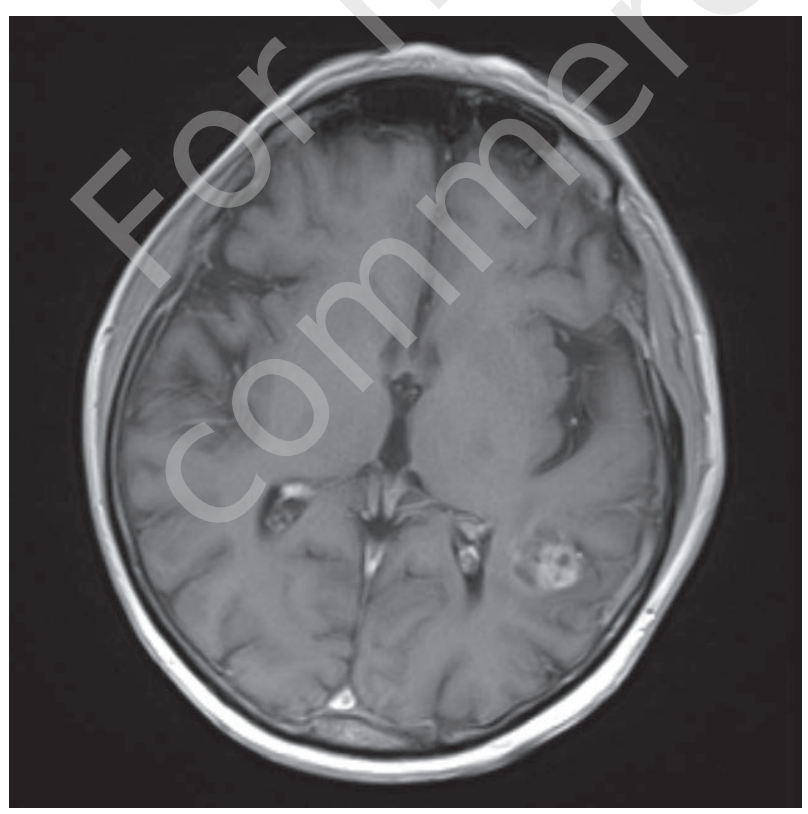

Taking into account the good general condition of the patient, the level of physical performance - WHO 1 and good tolerance of the current treatment, it was decided to use the II line of chemotherapy. Liposomal irinotecan in combination with 5-fluorouracil and leucovorin was included. Treatment commenced on December $5^{\text {th }}, 2019$ in limited doses. In the cardiologist's opinion, the patient was still at high risk of cardiological complications, but there were no absolute contraindications for oncological treatment. The ejection fraction was 33\%. G-CSF was used prophylactically.
In March 2019, a stabilization of the neoplastic process was found in the CT examination. CEA tumor marker concentrations decreased to $26.39 \mathrm{ng} / \mathrm{ml}$, and then to $16.3 \mathrm{ng} / \mathrm{ml}$, CA $19-9$ marker was $31 \mathrm{U} / \mathrm{ml}$ and $27.06 \mathrm{U} / \mathrm{ml}$ in May 2020. Morphology, bilirubin, AST, ALT and coagulation parameters returned to normal. The patient continued treatment without any significant symptoms of toxicity. Pain relief was also observed; since February 2020, the patient has not taken painkillers. He also continued low molecular weight heparin therapy. The patient remained under the care of a cardiologist who found no contraindications for further treatment. Another radiological evaluation was performed on June $5^{\text {th }}, 2020$. The CT scan showed regression of metastatic changes in the liver and stabilization of the tumor size in the pancreas (fig. 2). The patient received 11 courses of liposomal irinotecan in combination with 5-fluorouracil and leucovorin.

On June $24^{\text {th }}, 2020$, the patient was admitted with speech disorders in the form of motor aphasia to the Neurology Department of the Independent Public Psychiatric Healthcare Center in Choroszcz. MRI of the central nervous system (CNS) revealed a polycyclic, litho-cystic nodular mass of $21 \times 20 \times 22 \mathrm{~mm}$ surrounded by an edema zone in the left temporal region (fig. 3). The patient was qualified for stereotaxic radiotherapy of a tumor in the left parietal area in a total dose of $24 \mathrm{~Gy}$ in three fractions. The general condition of the patient deteriorated significantly (ECOG 4) since the diagnosis of CNS changes. The patient complained of weakness, pain in the abdominal cavity reappeared, and reported lack of appetite. He was qualified for symptomatic treatment and transferred to an inpatient hospice. He died on September $8^{\text {th }}, 2020$.

\section{DISCUSSION}

Treatment of pancreatic cancer is difficult. The lack of early warning symptoms, the rapid generalization of the neoplastic process and the deterioration of general performance make many patients ineligible for chemotherapy. Despite the constant search for new, effective methods of treatment, the results of treatment in pancreatic cancer patients remain unsatisfactory, and in most patients, due to the advanced stage of the disease, palliative chemotherapy remains the only treatment method at the time of diagnosis. 


\section{CONCLUSION}

This paper presents a case of a patient with advanced pancreatic cancer after surgery, in whom two lines of chemotherapy were used: gemcitabine in combination with nab-paclitaxel and liposomal irinotecan with 5-FU/LV. Treatment was well tolerated and most of the time on an outpatient basis.
Despite the palliative nature of the treatment and the presence of comorbidities (dilated cardiomyopathy with paroxysmal atrial fibrillation after chemotherapy for Hodgkin's disease 20 years ago), we managed to achieve a 2-year overall survival from the diagnosis of the disease.

\section{References}

1. Ducreux M, Cuhna A. Sa, Caramella C et al. Cancer of the pancreas: ESMO Clinical Practice.Guidelines for diagnosis, treatment and follow-up. Ann Oncol. 2015; 26(suppl 5): v56-v68.

2. Lambert A, Schwarz L, Borbath I et al. An update on treatment options for pancreatic adenocarcinoma. Adv Med Oncol. 2019; 11: 1143.

3. McGuigan A, Kelly P, Turkington RC et al. Pancreatic cancer: A review of clinical diagnosis, epidemiology, treatment and outcomes. World J Gastroenterol. 2018; 24(43): 4846-61.

4. Taieb J, Abdallah R. How I treat pancreatic cancer. ESMO Open. 2020; 4: e000818. https://doi.org/10.1136/.Esmo open-2020-0008.

5. Moore MJ, Goldstein D, Hamm J et al. Erlotinib plus gemcitabine compared with gemcitabine alone in patients with advanced pancreatic cancer: a phase III trial of the National Cancer Institute of Canada Clinical Trials Group. J Clin Oncol. 2007; 25: 1960-6.

6. Jassem J, Krzakowski M, Potemski P. Nowotwory układu pokarmowego. $2^{\text {nd }}$ ed. Via Medica, Gdańsk 2019.

7. Conroy T, Desseigne F, Ychou M et al. FOLFIRINOX versus Gemcitabine for Metastatic Pancreatic Cancer. N Engl J Med. 2011; 364: 1817-25.

8. Tempero MA, Malafa MP, Chiorean EG et al. Pancreatic adenocarcinoma, version 1.2021. NCCN Clinical Practice Guidelines in Oncology. J Natl Compr Canc Netw. 2019; 17(3): 202-10.

9. Tehfe M, Dowden S, Kennecke H et al. nab-Paclitaxel Plus Gemcitabine Versus Gemcitabine in Patients with Metastatic Pancreatic Adenocarcinoma: Canadian Subgroup Analysis of the Phase 3 MPACT Trial. Adv Ther. 2016; 33(5): 747-59.

10. Von Hoff DD, Ervin T, Arena FP et al. Increased survival in pancreatic cancer with nab-paclitaxel plus gemcitabine. N Engl J Med. 2013; 369: 1691-703.

11. Czyżykowski R, Janiak M, Krakowska M et al. Palliative systemic treatment of patients with pancreatic cancer - should reimbursement of nab-paclitaxel change the current management paradigm? Oncol Clin Pract. 2017; 13: 8-13.

12. Wang-Gillam A, Li C-P, Bodoky G et al. Nanoliposomal irinotecan with fluorouracil and folinic acid in metastatic pancreatic cancer after previous gemcitabine-based therapy (NAPOLI-1): a global, randomised, open-label, phase 3 trial. Lancet. 2016; 387: 545-57.

13. Klank-Sokołowska E, Kucharewicz M, Wojtukiewicz MZ. Nanoliposomalny irynotekan - nowe perspektywy leczenia uogólnionego raka trzustki. Onkol Prakt Klin. 2019; 5: 1-9.

Authors' contributions:

All authors contributed equally to the article.

Conflict of interests:

The authors declare no conflict of interest regarding the publication of this article.

Financial support:

None.

Ethics:

The authors had full access to the data and take full responsibility for its integrity.

All authors have read and agreed with the content of the manuscript as written.

The paper complies with the Helsinki Declaration, EU Directives and harmonized requirements for biomedical journals. 